\title{
A Solution For Improving TCP Performance Over Wireless Links
}

\author{
Ahmed Serhrouchni ${ }^{1}$, Adil Andaloussi ${ }^{1}{ }^{*}$, Abdel Obaid ${ }^{2}$ \\ 'ENST'Paris, CNRS URA 820 ; $^{2}$ Département d'informatique Université du Québec à Montréal
}

\begin{abstract}
The integration and the implementation of Internet transport protocols over wireless networks has increasingly proven to be unavoidable. However, the environments over which wireless protocols act are very different from those found on wired networks. Several contributions were proposed to adapt the current protocols such as TCP to the wireless environment. The goal is to improve the performance of TCP over hybrid networks (i.e. wired networks together with wireless ones) without modifying or violating the semantics of these transport protocols. In this paper, we look at the problem from a different perspective than the solutions that were proposed in the past and come out with an original approach. After its simulation, our new protocol called FACK-TCP (for Forced Acknowledgment TCP), proved to be very promising.
\end{abstract}

Keywords: Wireless TCP, Fack TCP, SNOOP, TCP performance.

\section{INTRODUCTION}

Nowadays, wireless communications are widespread on the WLAN level as well as on radio mobile network level. A great research effort is carried out to match these technologies with the Internet. The goal is to migrate towards an IP world. One of the challenges is to use TCP protocol in wireless communications in order to ensure a reliable end-to-end exchange of data.

TCP offers some mechanisms to handle congestion problems and it assumes that it is the main cause of packet loss in the Internet. In mobile networks however, packet loss is mainly due to the nature of the physical link and connection breakdown. If TCP is to be used in these networks, it will interpret these losses as a congestion problems. Which is not the case.

The original version of this chapter was revised: The copyright line was incorrect. This has been corrected. The Erratum to this chapter is available at DOI: 10.1007/978-0-387-35620-4_43 
There are several proposals to adapt TCP to wireless environments $[4,5]$. A possible classification of the solutions distinguishes :

- Solutions that attempt to extend standard TCP to the wireless communication environnement, trough the use of additional mechanisms and otions, or modification of the existing ones.

- Solutions that the require any modifications of the TCP/IP protocol stack. One approach is to isolate, in a transparent way, the wireless link from the rest of the network, this is what hybrid architectures aim at. Among these proposals we have Snooping-TCP [6,7] , Indirect-TCP [1] and WTCP [9].

- Other approches exist such as solutions application level solutions, or link layer solutions.

In the following sections, we state some of the problems and present some of the proposed solutions. We then tackle the main issue by introducing our solution, we call FACK-TCP (for Forced Acknowledgment TCP), its architecture and its behaviour. The breakthrough that we have achieved in this proposal is the introduction of a protocol which is both transparent to endpoints and easily deployable. Finally, we present some of our simulations results and compare them with Snooping-TCP and TCPReno.

\section{ADAPTATION OF TCP PROTOCOL TO RADIO MOBILE NETWORK}

TCP (described in RFC 793) [3] is the most used protocol of data transport over the Internet. Several variants were proposed as improvements over the original specification (e.g. Tahoe, Reno, Vegas) [2]. Several Internet applications such as FTP, Telnet and HTTP use TCP as transport protocol because of its performance and its reliability. Using this protocol on wireless networks without making any modification on the fixed host side will allow us to benefit from these existing applications.

Several approaches were proposed in using TCP in a mobile networks:

1. Split-connection strategy: Indirect-TCP [1] is one of the first protocols that uses this method. The idea is to split the TCP connection between the Fixed Host (FH) and the Mobile Host (MH) into two TCP connections with the Mobile Support Router (MSR) in middle. The MSR is a router located within a base station and its function is to acknowledge packets from a fixed host and to forward them to a mobile host. The advantage of this approach is to isolate flow and congestion problems of the wireless network from the fixed network. Also, transmission errors and packet losses on the wireless link do not propagate into the fixed network. Nevertheless this approach shows several drawbacks: 
- Loss of the end-to-end TCP semantics: Acknowledgements do not have an not end-to-end meaning. Acknowledgements packets may reach the fixed sender host before the packets actually reach the destination mobile host.

- Overhead: Every packet introduces an overhead because TCP packets are processed twice at the MSR and data must be copied at the MSR from the incoming TCP connection to the outgoing one.

2. The link layer mechanisms: These mechanisms are used to protect fixed host [2]. Two mechanisms are used in this approach:

- ARQ (Automatic Repeat reQuest) whereby the information is segmented into packets to which are attached error checking sequences (usually through $\mathrm{CRC}$ computing). This allows the receiver to detect corrupted packets and ask for retransmission.

- FEC (Forward Error Correction) in which redundant blocks are added to the information packets before they are sent. These blocks allow for error detection and correction at the receiving side.

Notice that most of the time, upper layer protocols provide end-toend reliability so that it is redundant to implement sophisticated error correction procedures within link layer protocols.

3. TCP end-to-end improvements: The idea is to try to improve the TCP fast retransmission mechanism in order to be able to handle the multiple losses in a same window without necessarily pipe exhaustion as it is the case with Fast Retransmit and Fast Recovery. Several improvements to TCP are based on this idea:

- NACK (Negative Acknowledgement): In this approach, an additional negative acknowledgement is added in the option field of the TCP header in order to indicate which packet is received with an error and to perform the retransmission of this packet as soon as possible, particularly if several packets were corrupted in the same window.

-SACK (Selective Acknowledgement): In this approach, the receiver stores up to the last three data packets that were received correctly and sends back the cumulative acknowledgment to the sender, which can then determine which packets to retransmit.

4. Explicit notifications: This approach [8] allows the receiver or any intermediary node on the path to warn the sender of the nature of a loss (congestion, corruption, interruption) that has occurred, or that is likely to occur. The sender can then react adequately as opposed to standard TCP, where loss signals are not direct notifications, and are interpreted as due to congestion. Two mechanisms are used in 
this approach: Explicit Congestion Notification (or ECN) and Explicit Loss Notification (or ELN). The use of explicit notifications implies the modification of TCP at the level of the sender and the modification of IP/ICMP at the level of the sender, the receiver and the intermediary nodes, which is clearly heavy.

5. Wireless protocols: These protocols are matched to the TCP protocol in order to avoid some of the problems encountered with the wireless part of a connection. The main idea of these protocols is to deploy an agent within the base stations which will recover from any lost packets by performing local retransmissions. They modify the link software in the base stations and the transport software in the mobile stations. The advantage of these protocols is that they require no modification to TCP implementations in the fixed network (Figure 1).

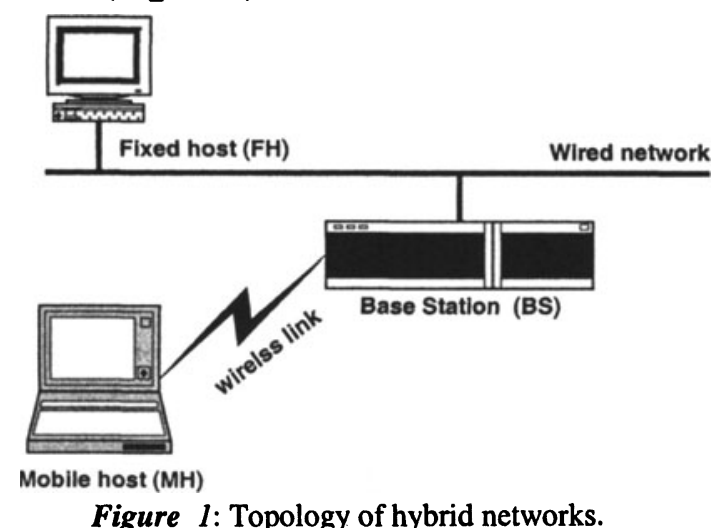

In the following sections, we will discuss one of these protocols, namely Snooping-TCP.

\section{THE SNOOPING-TCP PROTOCOL}

In Snooping-TCP [6,7], an agent called Snoop agent is added at the network level in the base station (BS). It handles the packets and their corresponding backward acknowledgments that are exchanged between the fixed host (FH) and the mobile host (MH).

For data transfer from $\mathrm{FH}$ to $\mathrm{MH}$, the snoop agent maintains a cache where it stores all new packets that arrive in sequence, which it forwards to the $\mathrm{MH}$. When a packet is received that has been cached earlier, then either the packet has not been acknowledged to the fixed sender and it is simply forwarded to the $\mathrm{MH}$, or it has been acknowledged and then a new ACK is sent to the fixed sender. When an out-of-order (non cached) packet is received, it is simply forwarded to the $\mathrm{MH}$, with a tag indicating that it has been retransmitted by the sender. When the agent receives a new ACK, the 
acknowledged TCP bulk data packets are freed from the cache, and the ACK is forwarded to the FH. If the ACK is a duplicate ACK for a packet not cached nor tagged as retransmitted from the sender, it is forwarded to the fixed sender, so as to possibly trigger congestion control mechanisms. If the ACK is a duplicate ACK for a cached packet, it is retransmitted via a priority queue.

For data transfer from $\mathrm{MH}$ to $\mathrm{FH}$, the snoop agent uses the ELN mechanism. The agent detects the segments altered over wireless link and when it receives duplicate acknowledgements for these segments, it notifies the $\mathrm{MH}$ that the loss is due to corruption in wireless link. This way, the $\mathrm{MH}$ doesn't call upon the congestion control mechanisms.

These mechanisms together improve the performance of the connection in both directions without loosing any of the end-to-end semantics of TCP or modifying host TCP implementation in the fixed host.

Despite the fact that it improves the TCP performance, Snooping-TCP has several drawbacks. First, it does not manage long interruptions in connections due to handovers. Secondly, the packets could be retransmitted by the agent and the protocol cannot avoid the expiry timer of the FH from being triggered. In addition, the cache can cause a congestion in the gateway. Lastly, it is necessary to take into account of the big overhead generated by this protocol. The update of the memory cache is executed for each packet or acknowledgement reception and the management of this memory consumes a lot of CPU. This penalizes the overall throughput especially when the radio channel quality is good.

\section{THE FACK-TCP PROTOCOL}

The idea behind FACK-TCP is to control the sender's fixed host by using Fast Retransmit mechanism. A FACK-TCP module installed in base station forwards the packets without caching them. The module records the last acknowledgement sent by the mobile and sets a local timer for new packet arrival. If a packet is lost, FACK-TCP will not receive an acknowledgment and the local timer will expire. In this case, the module sends 2 acknowledgment packets to the fixed host to force it to enter into the FastRetransmit mode before its timer expires. This enables us to avoid bringing the connection into the slow start mode and to maintain a satisfactory throughput.

We install a FACK-TCP module on the base station. This module forwards each packet to the mobile without cashing it and startsa local timer. The module keeps track the last acknowledgment sent by the mobile, called Last_ACK. If the acknowledgment of a packet is not received in RTO (Round Time Out), FACK-TCP sends Last_ACK two times back to the FH. 
When the fixed host receives these acknowledgments, it enters the FastRetransmit mode. This way, we ensure that TCP does not go into Slow start mode, which could have reduced the overall throughput.

We can decompose the FACK-TCP module into 3 parts:

- Reception of packets coming from FH

- Reception of acknowledgements from MH

- Local timer expiry

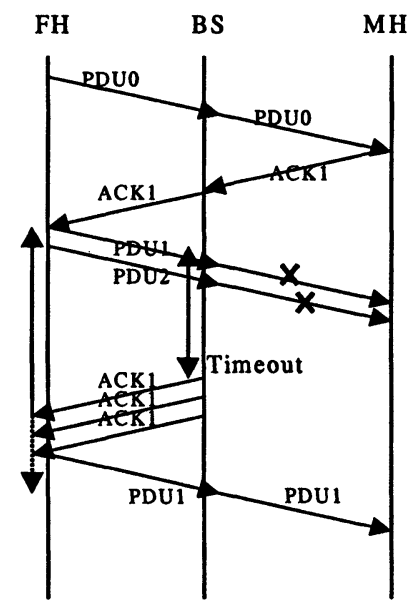

Figure 2: A FACK-TCP exchange.

Reception of packet coming from $\mathrm{FH}$

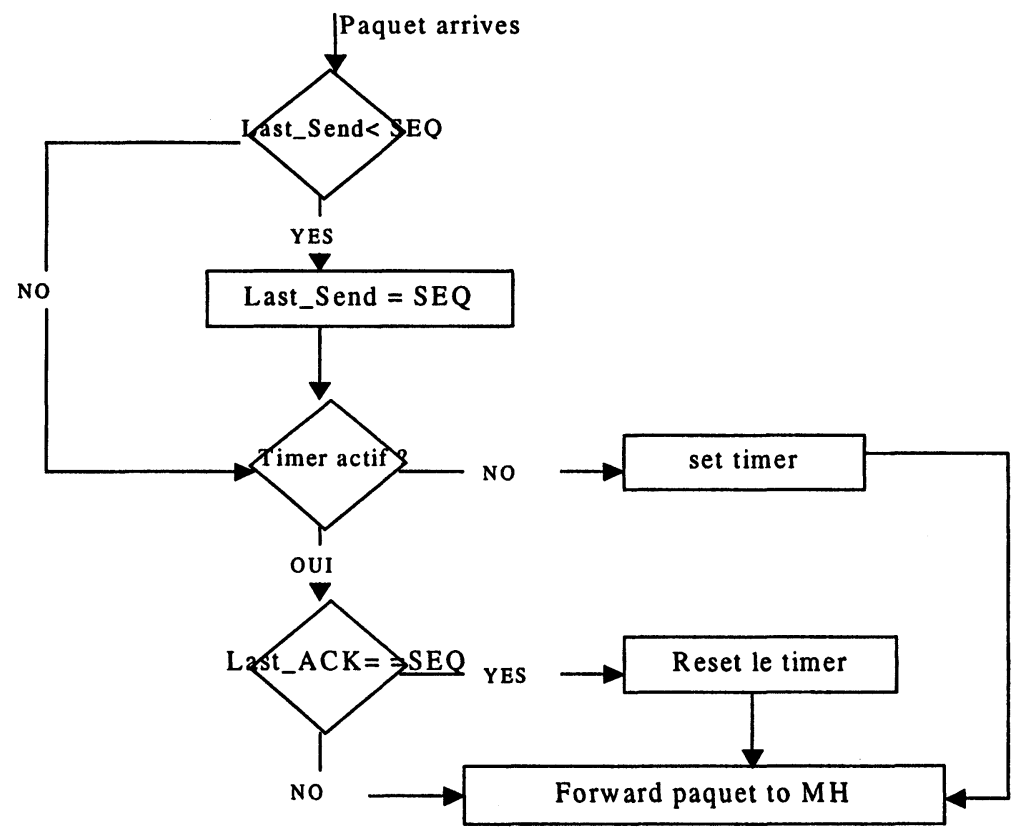

Figure 3 : Reception packet from $\mathrm{FH}$. 
The FACK-TCP module keeps a variable, called LAST_SEND, which corresponds to the sequence number of the last message sent from the base station. This will enable the base station to determine whether a packet was acknowledged or not. A variable called $S E Q$ corresponds to the sequence number of the current packet and $L A S T \_A C K$ corresponds to the last ACK received. The module sets a timer after the arrival of a packet unless there exists in the wireless network a different un-acknowledged packet.

\section{Reception acknowledgement from $\mathbf{M H}$}

Variable $A C K$ corresponds to the acknowledgement number and variable $N A D$ corresponds to the number of times the module must send $L A S T \_A C K$ to FH once the timer expires. The module checks if the acknowledgement is duplicated. If so, the module updates NAD otherwise $L A S T$ _ACK becomes equal to $A C K$ and the local timer is stopped. Note that the timer will be stopped only if there is no other packets waiting to be acknowledged. Otherwise, it will be reset.

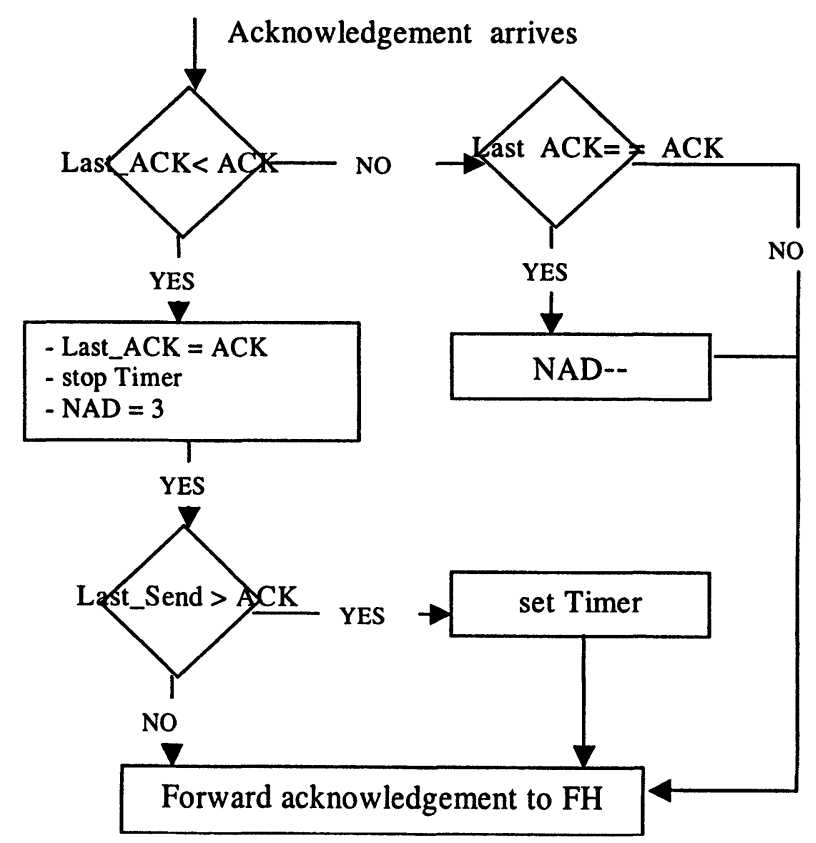

Figure 4 : Acknowledgement from $\mathrm{MH}$

When the local timer expires, FACK-TCP sends $L A S T \_A C K$ a number of times equal to $N A D$ back to FH. This will start Fast-Retransmit and retransmits the lost packets by decreasing the threshold of congestion control. 


\section{PERFORMANCE}

We have implemented FACK-TCP and tested it with NS (Network Simulator) software. We have simulated the protocol with different bit error rates (BER) and compared the results with Snooping-TCP and TCP-Reno. Figure 5 shows our simulation architecture. We used a wired LAN instead of a wireless network because the version of Snooping-TCP which is currently available on NS works only within a local area network. We used the characteristics of a LAN with a throughput of $1.5 \mathrm{Mb} / \mathrm{s}$ and a delay of $10 \mathrm{~ms}$.

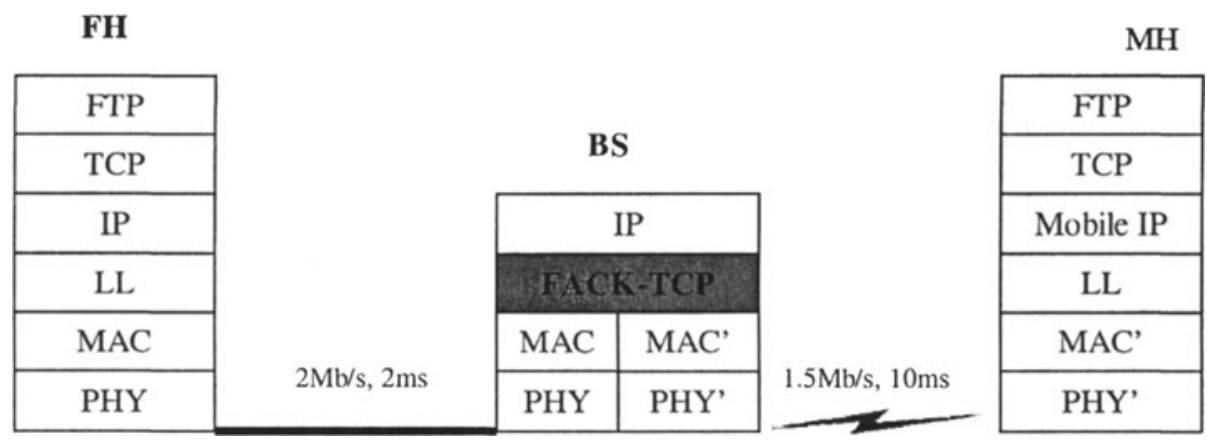

Figure 5 : Simulation architecture

Using an FTP flow connection between $\mathrm{FH}$ and $\mathrm{MH}$, we obtained rather interesting results. In the presence of no packet loss, the maximum throughput achieved by a TCP connection was about $1.28 \mathrm{Mbits} / \mathrm{s}$. The sender TCP stack was based on TCP Reno (an implementation supporting Fast-Retransmit after reception of three duplicate acknowledgements). The maximum window size for the connection was 40 packets and the maximum TCP segment size was 1000 bytes. In order to measure the performance of FACK-TCP, we used a uniform distributed bit error model. To do so, we installed an error generator in the LAN, which altered some packet's TCP Checksum so that the receiver drop some (corrupted) packets.

Figure 6 presents the results of the simulation of three connections with bit $B E R$ varying between $1.5^{*} 10^{-5}$ and $2.5 * 10^{-7}$. The first connection implements the Snooping-TCP, the second uses FACK-TCP while the third uses TCP-Reno only. The duration of each simulation is $1000 \mathrm{~s}$. In the figure, the vertical axis represents the throughput of each connection, while the $\mathrm{X}$-axis indicates the inverse of bit error rate (1/BER). For each BER, we took the average of the throughput obtained in 20 simulations.

We could see that the FACK-TCP protocol performs significantly better than TCP-Reno for a given BER. With FACK-TCP, we remarkably increased the throughput while significantly decreasing the number of times 
FH had to go into slow start (85\%). FACK-TCP is more efficient than Snooping-TCP for small and medium BER. The diagram shows a linear increase for FACK-TCP over Snooping-TCP for BER intervals which are close to real life situations. On the other hand, the throughput reached with Snooping-TCP is rather constant on this interval. In fact, Snooping-TCP carries out a clearer performance when the BER is high and decreases the number of times the $\mathrm{FH}$ timer expires by $50 \%$.

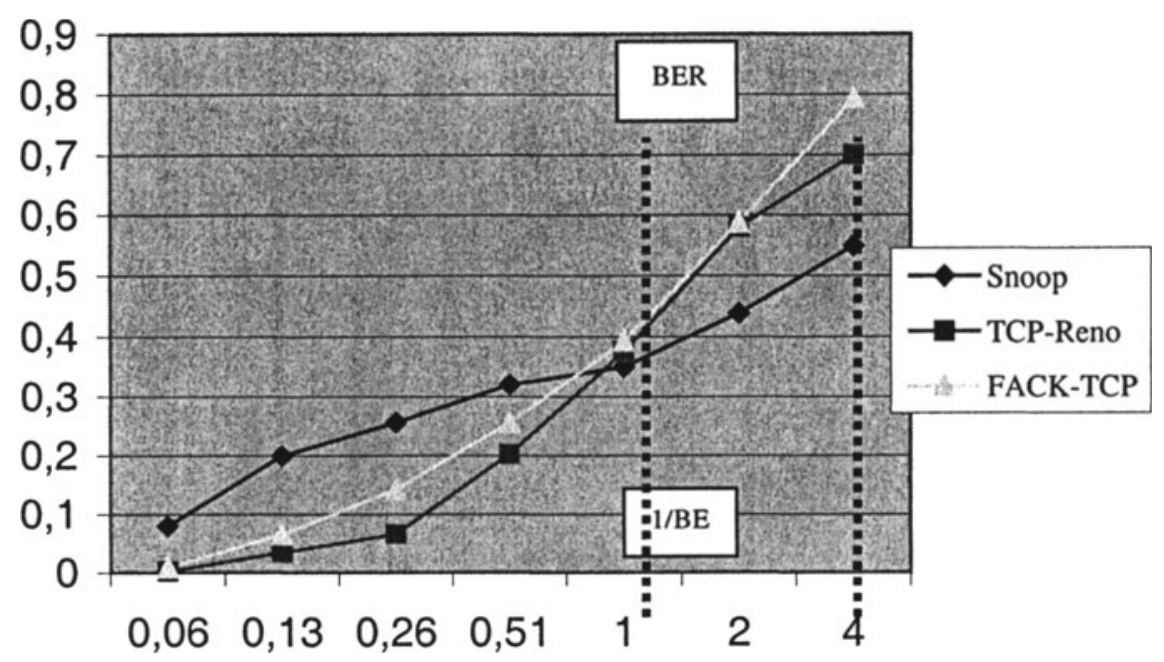

Figure 6 : The throughput obtained with 1/BER

When BER is less than $10^{-7}$, we see a clear decrease in performance for Snooping-TCP compared to the two other protocols. This is mainly due to the overhead caused by the Snooping-TCP module. With each reception of a packet, the module carries out several loops to insert it in its memory before transmitting it to the other end. And with each reception of an ACK, it traverses the memory all over again to remove all the acknowledged packets. This process generates a heavy processing which consumes much of CPU time.

Figures 7 and 8 show the results of two simulations which compare the sequence number evolution of TCP segments emitted by FH in three connections, with two different bit error rates: $3.9 * 10^{-6}$ and $10^{-6}$.

The evolution of sequence number using FACK-TCP with a BER equal to $3.9 * 10^{-6}$ is more significant than with TCP-Reno. Yet, it is less important when using the Snooping-TCP protocol. With FACK-TCP, the throughput obtained is equal to $0.142 \mathrm{Mb} / \mathrm{s}$. With Snooping-TCP the throughput is equal to $0.26 \mathrm{Mb} / \mathrm{s}$ and with TCP-Reno we obtained $0.065 \mathrm{Mb} / \mathrm{s}$. We can then see that the performance of Snooping-TCP decreases when its buffer cannot hide 
packets anymore. When a BER is equal to $10^{-6}$, we observe a slight improvement of FACK-TCP performance over the other protocols. Its throughput was equal to $0.393 \mathrm{Mb} / \mathrm{s}$, while with Snooping-TCP and TCPReno, we obtain $0.368 \mathrm{Mb} / \mathrm{s}$ and $0.373 \mathrm{Mb} / \mathrm{s}$ respectively.

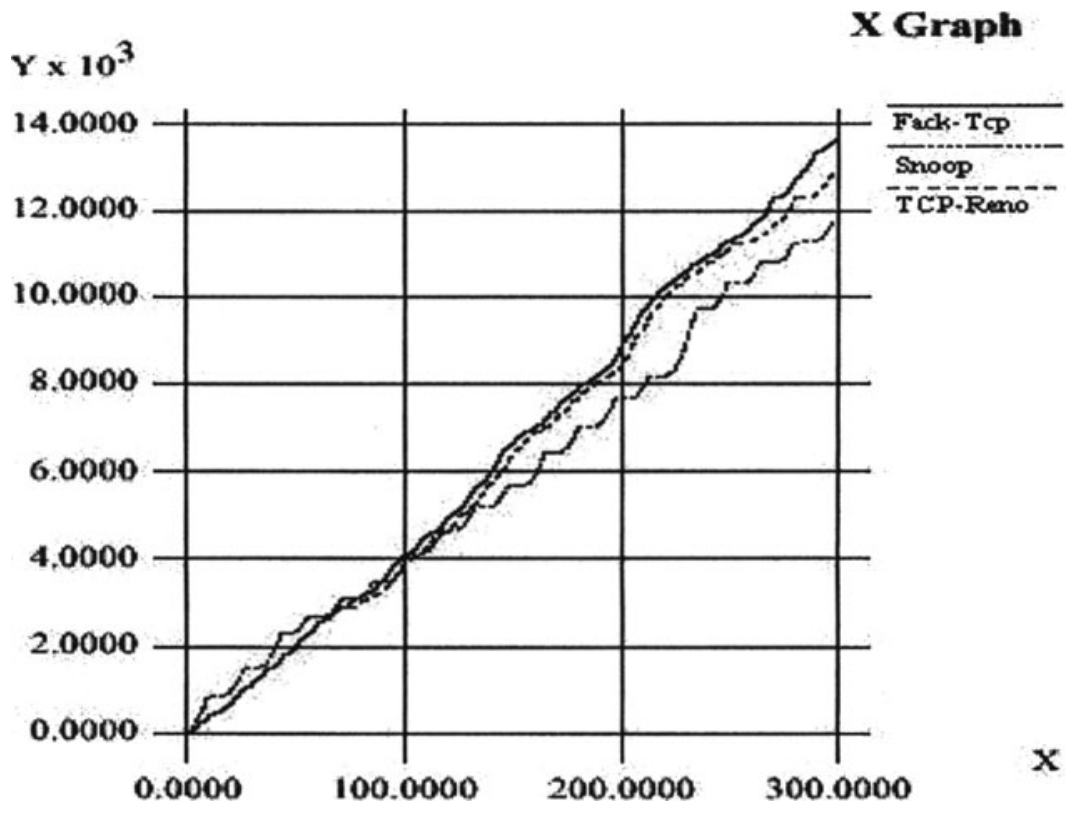

Figure 7: Evolution of sequence numbers $\left(\mathrm{BER}=3.910^{-6}(1 / 256 \mathrm{~kb})\right)$.

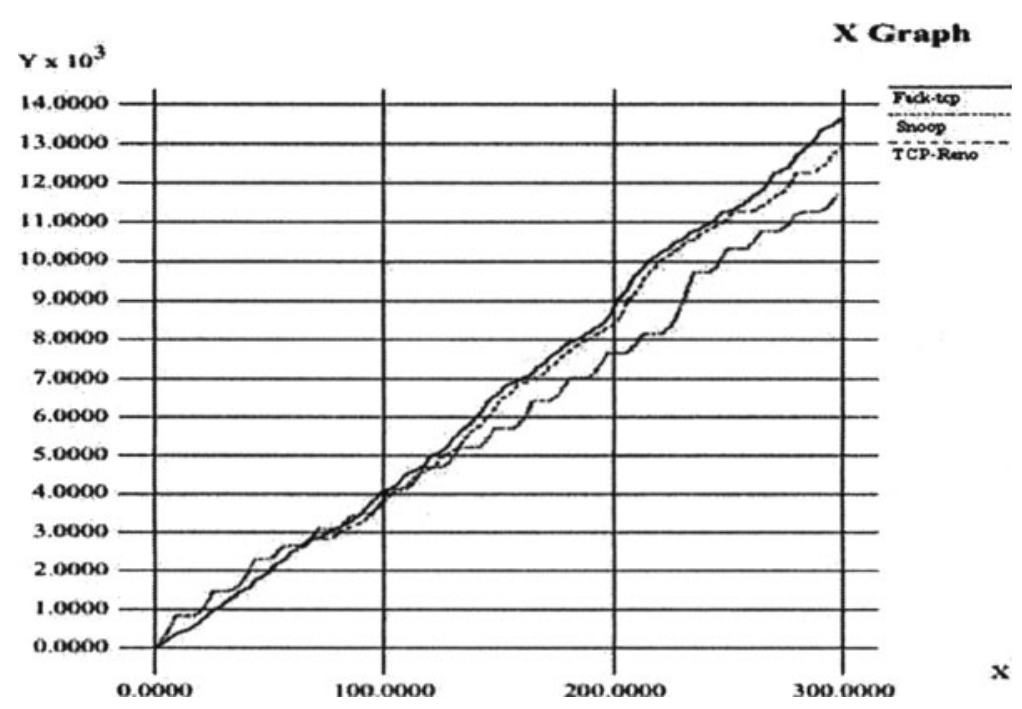

Figure 8: The evolution of sequence number to $\mathrm{MH}$ with a $\mathrm{BER}=10^{-6}(1 / 1 \mathrm{Mb})$ 


\section{CONCLUSION}

In this paper, we presented different problems and the solutions for TCP over wireless network. Most of these propositions increase the TCP performance but involve modifications in the existing TCP implementations and then could cause interoperability problems.

The main advantage of Snooping-TCP is to be completely transparent to the fixed host. The protocol significantly improves the TCP performance. However, it has several drawbacks as we explained in this paper. It is the main motivations why we came up with a FACK-TCP protocol.

The objectives that we have set with FACK-TCP are :

- Transparency to the mobile and fixed side with not sacrifying the TCP semantic.

- Less overhead and simplicity in implementation

- Less overload of the mobile host

The simulation results showed that FACK-TCP is more efficient than both Snooping-TCP and TCP-Reno for a given bit error rate interval. FACKTCP protect (in a way or another) the fixed host from long interruptions in wireless network (due to handover). The performances of FACK-TCP protocol are certainly lower than those of Snooping-TCP when the radio channel quality is very bad. But for low bit erraor rates, FACK-TCP is a suitable thanks to its simplicity.

We think that in the future, we will see the use of a combination of both Snooping-TCP and FACK-TCP protocols with mechanisms to switch between the two protocols according to observed BER and gateway overload.

\section{REFERENCES}

[1] Bakre, A., Badrinath, B.: ' I-TCP : Indirect TCP for mobile hists', Proc. of the $15^{\text {th }}$ International Conference on Distributed Computing Systems (ICDCS), Vancouver, Canada 1995.

[2] A. Serhrouchni : 'Problèmes et solutions pour TCP sur Satellite' , Course, ENST-Paris, 1997.

[3] W.R. Stevens - 'TCP/IP illustré', Volume 1. Vuibert, 1998.

[4] R.Cacers and L.Iftode. 'Improving the Performance of Reliable Transport Protocols in Mobile Computing Environments'. IEEE Journal on Selected Areas in Communications, 13(5), June 1995.

[5] R.Cacers and L.Iftode. 'Improving the Performance of Reliable Transport Protocols in Mobile Computing Environments'. IEEE Journal on Selected Areas in Communications, 13(5), June 1995.

[6] Hari Balakrishnan. 'Challenges to Reliable Data Transport over Heterogeneous Wireless Networks'. PhD Thesis, University of California at Berkeley, 1998. 
[7] H. Balakrishnan et al. 'A Comparison of Mechanisms for Improving TCP Performance over Wireless Links'. In Proceeding of ACM SIGCOMM'96, August 1996.

[8] S. Floyd. 'TCP and Explicit Congestion Notification'. Computer Communication Review, 24(5), October 1994.

[9] Karu Ratnam and Ibrahim Matta. "WTCP: An Efficient Mechanism for Improving TCP Performance over Wireless Links". Third IEEE Symposium on Computer and Communications (ISCC '98). 